\title{
DTN Implementation and Utilization Options on the International Space Station
}

Kelvin Nichols

Mark Holbrook

Lee Pitts

Kevin Gifford

Andrew Jenkins

Sebastian Kuzminsky 


\section{Outline}

$\checkmark$ Brief introduction of the current communications architecture of the ISS

- How current payload operations are handled in the nonDTN environment

- Making the case to implement DTN into the current payload science operations model

- Phase I DTN Operations: early implementation with BioServe's CGBA Payload

- Phase II DTN Operations: Developing the HOSC DTN Gateway

$\bullet$ Conclusion 


\section{Introduction}

ISS Supporting Ground system

- Space links are based on CCSDS silver standard

- S-band used for low bandwidth Uplink/downlink

+ Primary payload command uplink path

+ Approximate uplink rate of $38 \mathrm{Kbps}$

$\checkmark$ Ku-band used for high bandwidth Telemetry downlink

+ Primary downlink path for payload telemetry

+ Approximate downlink rate of $50 \mathrm{Mbps}$

- Harsh environment of space presents a new set of problems over traditional IP networks

+ AOS/LOS scheduling issues

+ Radiation

+ Sharing downlink with other spacecraft

$\checkmark$ Ground transport is over IP networks

+ Payload data is distributed to users via UDP

+ Remote commanding is encrypted over VPN
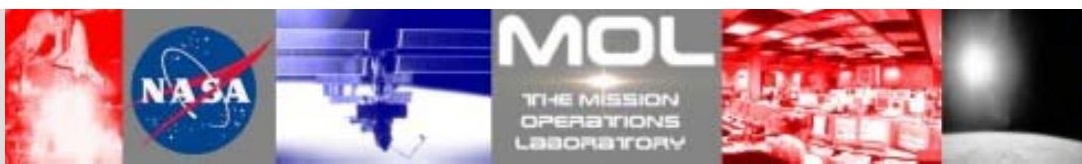


\section{Introduction}

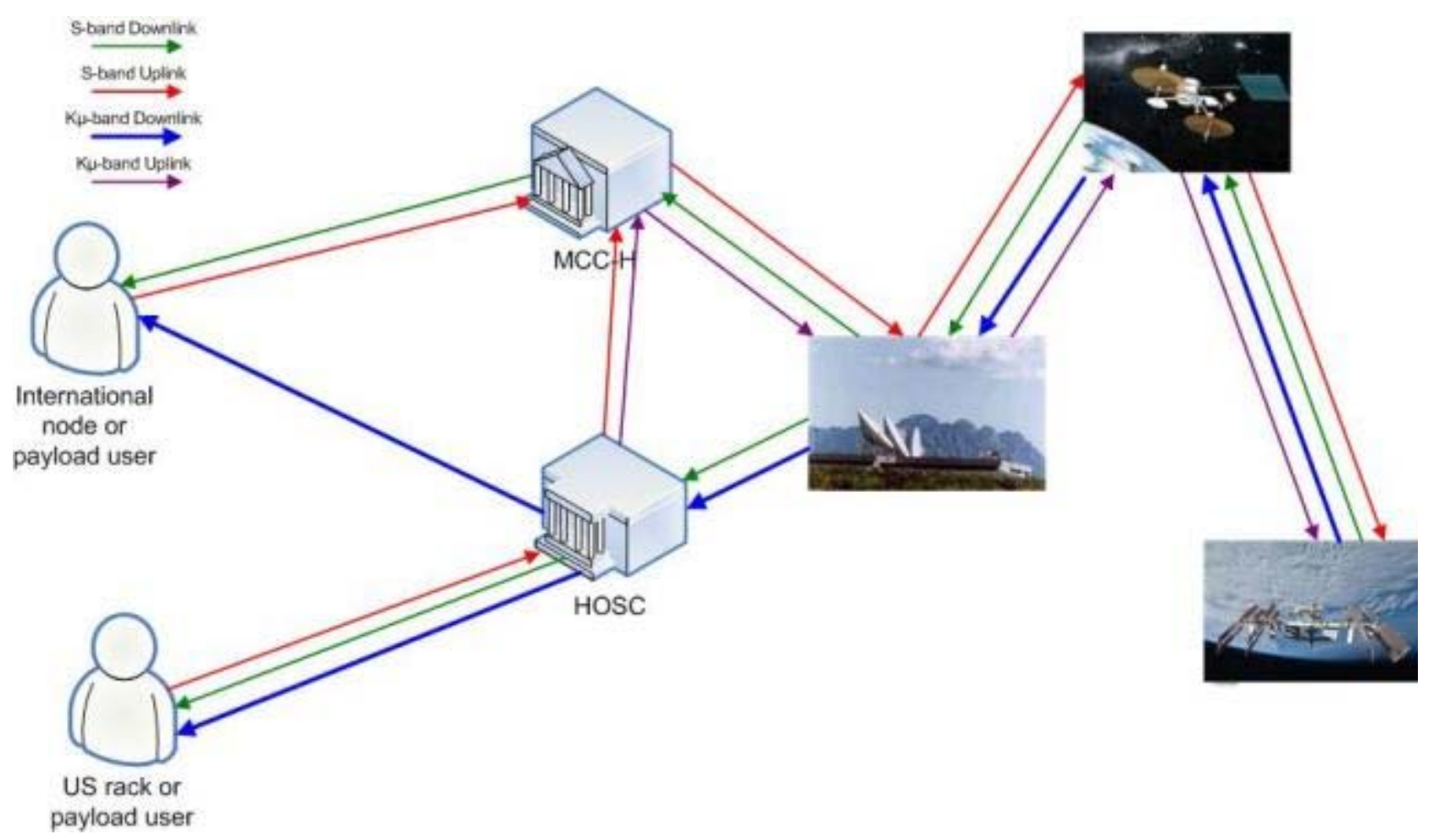




\section{The non-DTN environment}

Primary function of the HOSC

- to support Payload Investigators and the corresponding science

+ Tools and protocols have been utilized so PI can command and receive data nay place in the world

$\diamond \mathrm{UDP} / \mathrm{TCP}$

$\diamond$ HTTPS

$\diamond \mathrm{RSH}$

$\diamond$ FTP

- Also support Command and Control applications for Payload Investigators

+ TCP based and wrapped in secure envelope of IPSEC compliant VPN

- The C\&C and Payload science distribution are handled in parallel

- Science distributed through PDSS via UDP

- Down-linked CCSDS data is constrained to it's max size

- EHS header pre-pended to expedite processing

- Users can request missed data through a user scheduled playback

- Science distributed through PDSS via UDP

- Down-linked CCSDS data is constrained to it's max size

- EHS header pre-pended to expedite processing

- Users can request missed data through a user scheduled playback
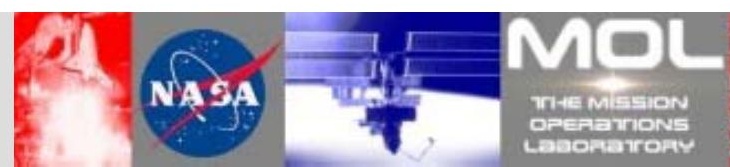


\section{Making the case to implement DTN}

DTN would greatly help with the issues related to scheduled around planned communication outages

- Several important considerations were part of the design

- Wanted to support the needs of our ever changing user base

$\checkmark$ Current HOSC model works best with a control center on a highly available network

+ However the HOSC has many smaller centers and experiments that don't want to staff continuously

+ A DTN environment would better suit a type of on demand service

$\diamond$ User would not have to be online $100 \%$ of the time to receive data

$\diamond$ Would just pick it up the next time he/she logs into the system

- Also user would not need to be up all the time for commanding either

+ User could log in once with a set of commands that needed to be sent, then log off and be assured that they would be delivered onboard when a window was available
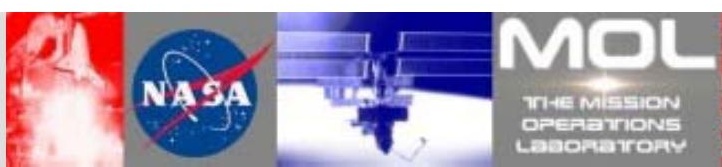


\section{Phase I DTN Operations:}

- The HOSC partnered with CU-Boulder's to create the first path finder DTN experiment onboard ISS

- CU-Boulder was able to repurpose it's CGBA (Commercial Generic Bioprocess Apparatus) experiment and install the ION version of DTN code

- The HOSC developed a DTN command queue to allow Cadre management of DTN acknowledgments

+ This is not really a DTN node but more like a priority queue

+ When opportunity presents itself on commanding link, these acknowledgments would then be forwarded through JSC commanding to go back up to CGBA payload to confirm ground receipt

- This style of special queued commands was different from the normal operations concept used in the POIC

- Had to keep ground station operators informed during this phase to allow them to be ready for the new way DTN commanding would be allowed to circumvent the traditional commanding 


\section{DTN I Early results:}

- The modified ground software was deployed in the April time frame of 2009

- First DTN experiments from CGBA followed in the June 2009 timeframe

+ First experiments involved down-linking pictures from a previous CGBA experiment.

+ More extensive test were performed after this first round of experimentation was successful

- The new experiments were to test how the payload would respond to unattended operations

+ Status telemetry files were down linked using non-DTN and DTN paths

$\diamond$ Non DTN scheme yielded an average of 3504 redundant receptions per file

$\diamond$ DTN scheme yielded an average of 0.06 redundant receptions per file

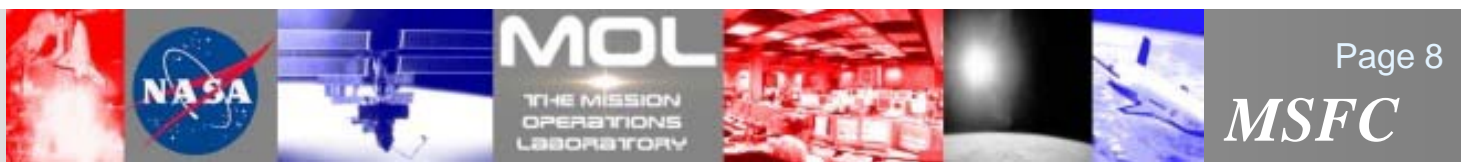




\section{DTN Phase I: Implementation}

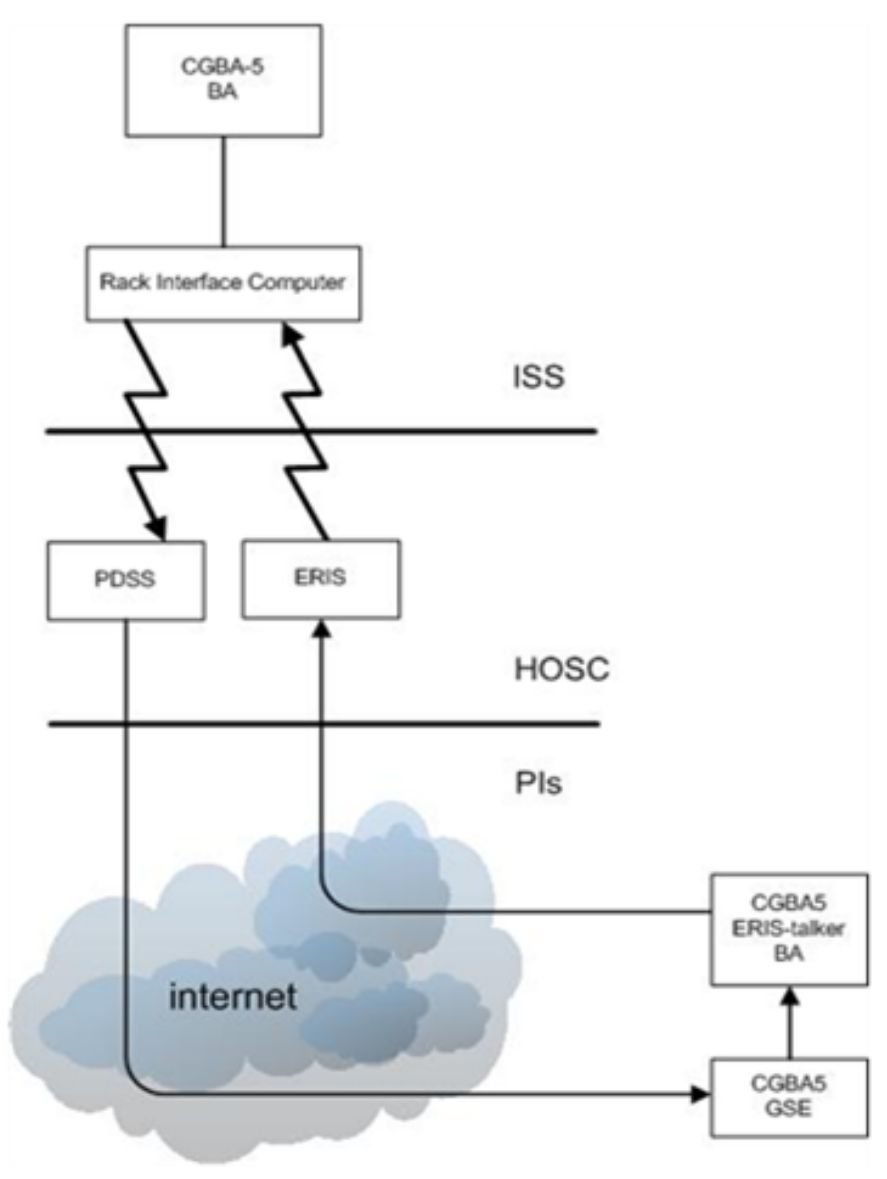

- CU-Boulder sends a bundle encapsulated in a command to ERIS listenter on an ePVT server.

- ERIS forwards the command to the command server and the DTN command queue.

- After CSM requirements are met the command is forwarded to JSC and ultimately CGBA-5 on ISS.

- Acknowledgements are forwarded via Telemetry and Ku-Band

- Downlink science bundles are sent in CCSDS packets via Ku-Band to PDSS which forwards the data to CGBA-5.

- Acknowledgements to CGBA-5 are forwarded via S-band.

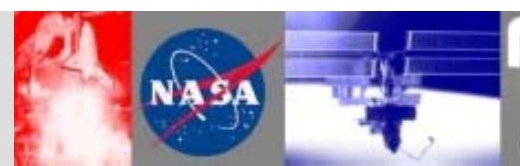




\section{Phase II DTN Operations:}

- HOSC is in the process of developing it's own DTN node using a phased approach:

- Initial development and evaluation

- DTN Engineering Network (DEN) testing

- DEN testing with CGBA using recorded data

- IV\&V testing with recorded and live data

- Operational data flow 


\section{Phase II DTN Operations:}

- Initial development was performed using DTN2 instance

- Testing with the CGBA ION DTN implementation uncovered some issues

+ Related to ION and DTN2 interoperability

+ Also related to some unique ways that communications are implemented onboard the ISS

- To handle these issues a Convergence Layer Adaptor (CLA) was implemented unique to the ISS

+ The CLA identifies and extracts the embedded BioServe RIC channel packets and then extracts the BP bundle set

+ The packets are then processed by the DTN2 daemon for bundle separation, forwarding, custody transfers, and any other processing 
IV\&V Data Flow

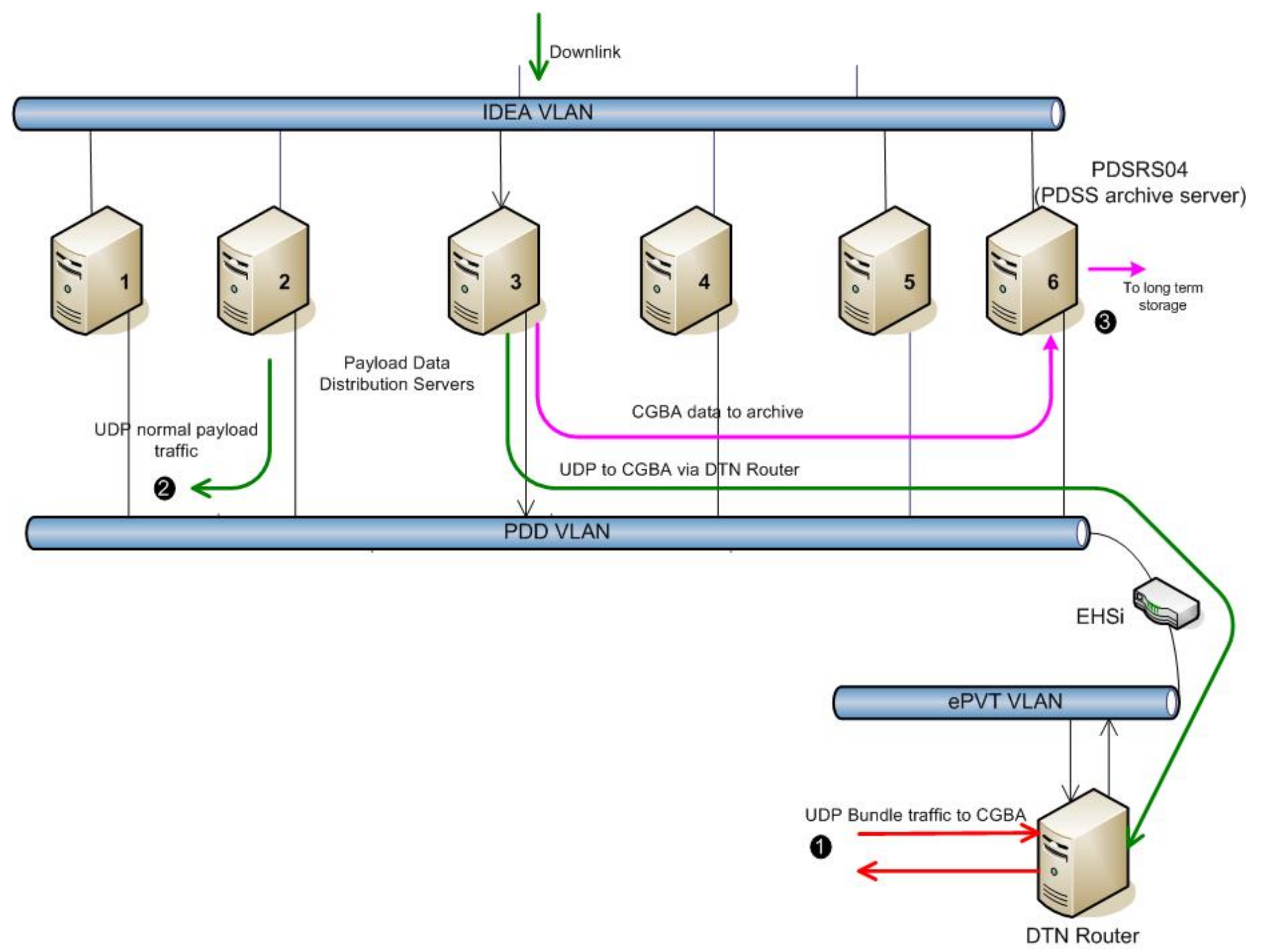




\section{High Available DTN configuration}

- It will support multiple routers in a prime and backup mode

The use of shared Redundant Array of Independent Disk (RAID)

$\checkmark$ Will allow HOSC to support End-to-End DTN traffic from CGBA

- Configuration also preserves the separation science Downlink and S-Band commanding 
Highly Available DTN Config

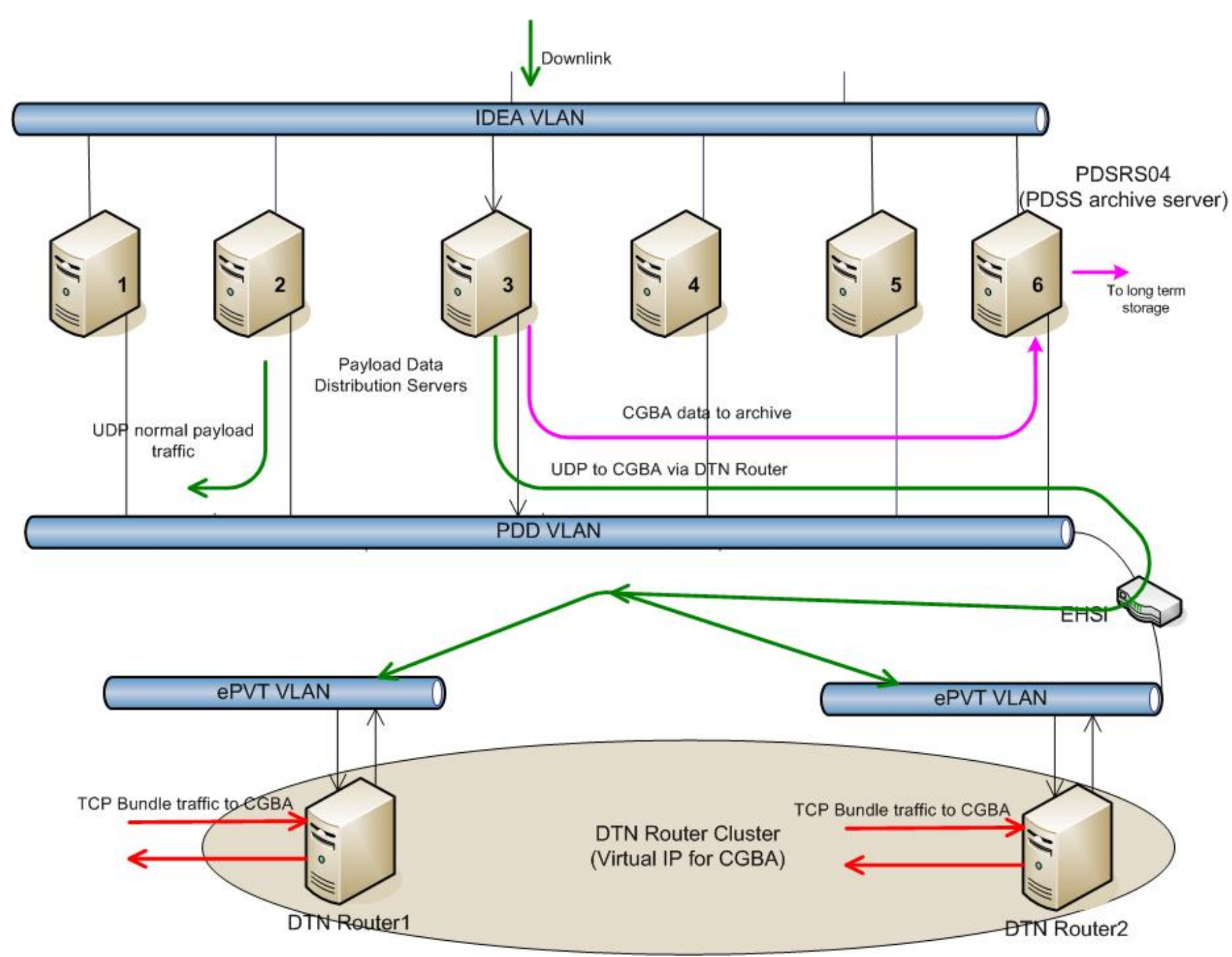




\section{Future DTN work}

- The next goal is to break the separation described on the previous slide.

- This will be accomplished it 2 phases:

- Encapsulate the Application Identifier (APID) and forward with custody transfer

- Pull out the bundle and perform a custody transfer

$\checkmark$ Option 2 is preferred and the direction the HOSC is pursuing 


\section{Option 1}
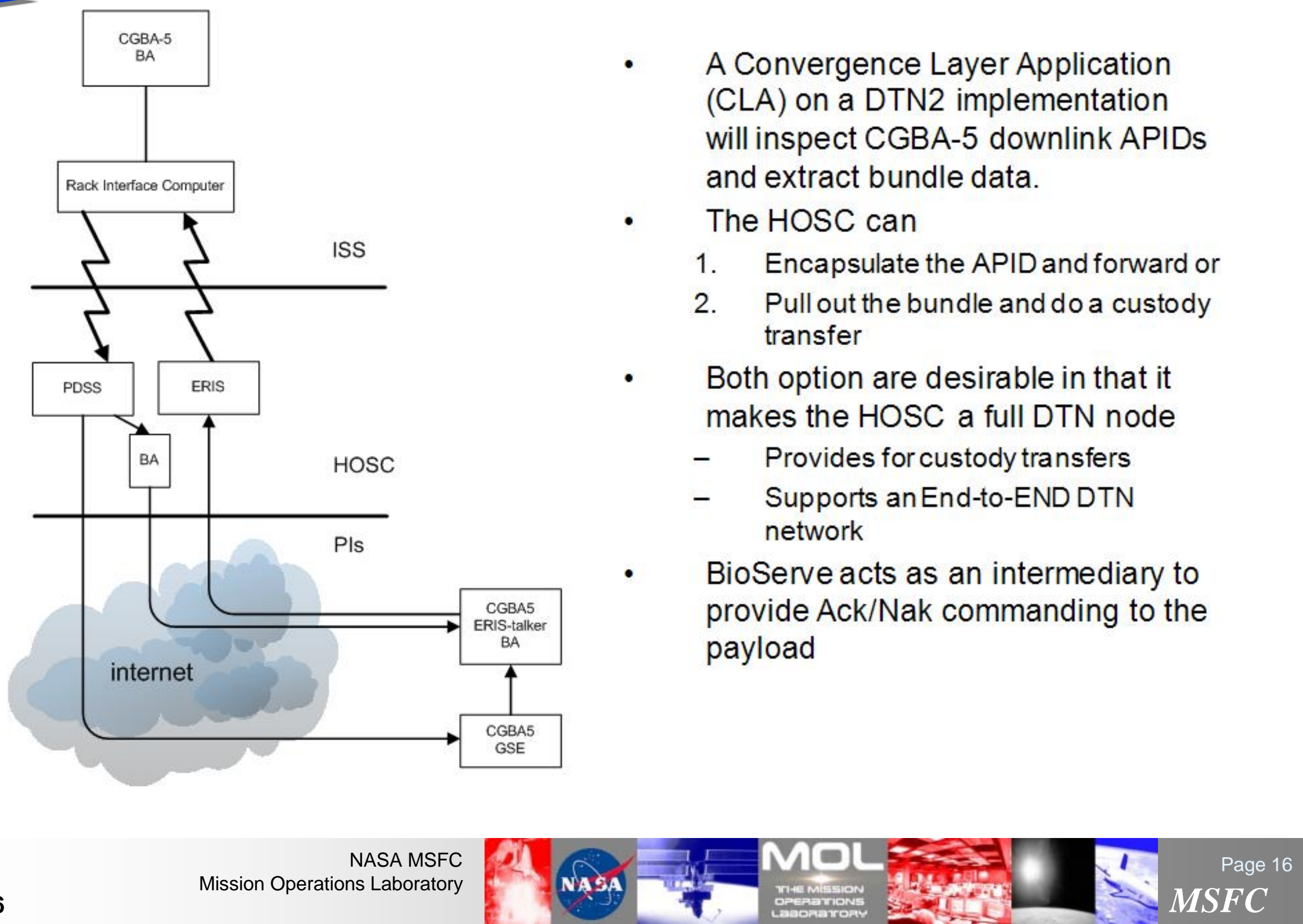


\section{Option 2}
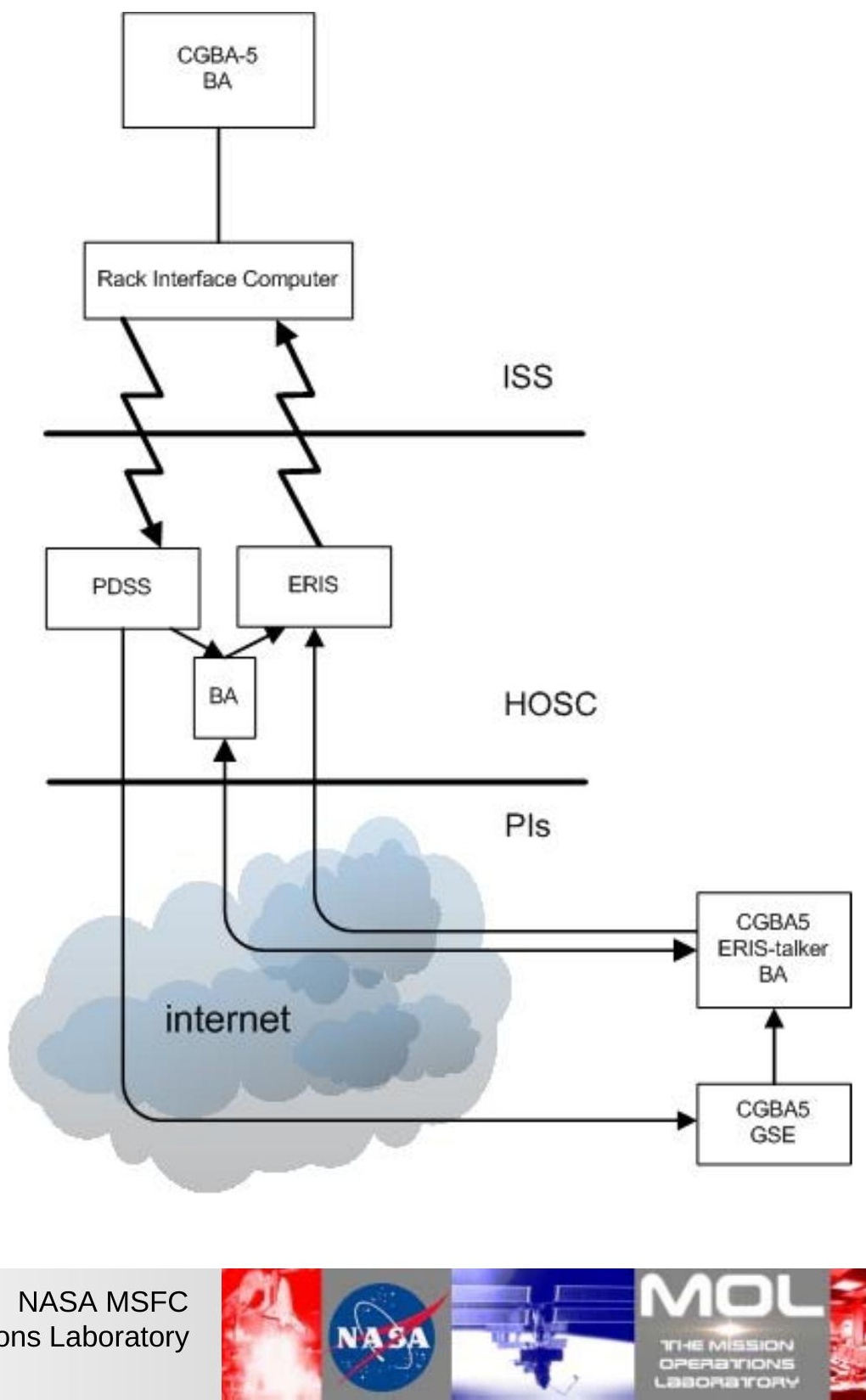
Integrated Config to support both DTN and NonDTN users
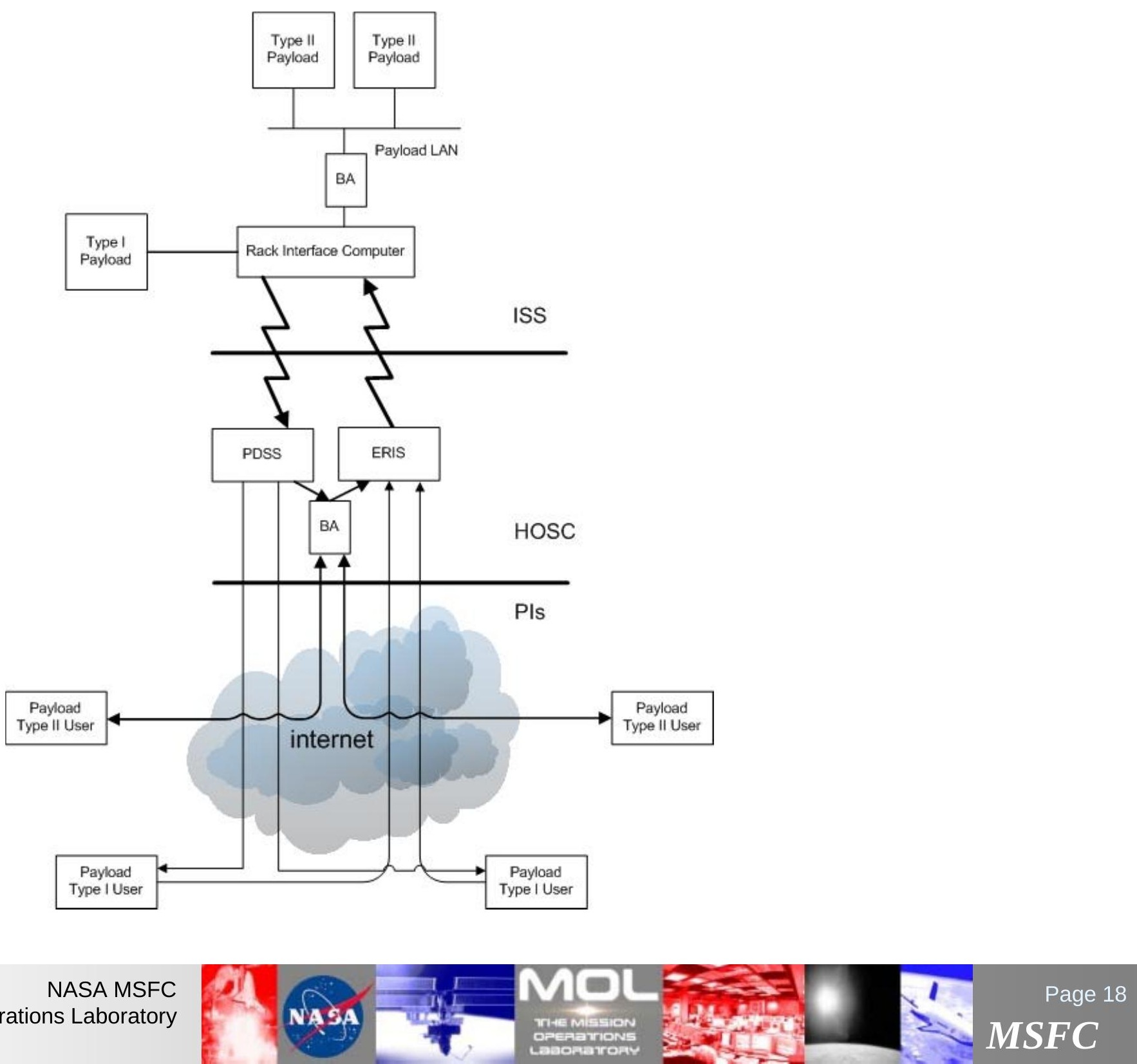


\section{Conclusion}

Allows more diverse options for users

- Supports new and innovative operations concepts

- Leverages ISS as a test bed for new initiative

- Can support various users

- Continues the evolution of the ISS 